\section{Maximal stability limits in adolescents with Tourette syndrome}

\author{
Mariève Blanchet ${ }^{1 *}$, François Prince ${ }^{2}$, Martin Lemay ${ }^{1}$, Sylvain \\ Chouinard ${ }^{3}$ and Julie Messier ${ }^{4}$
}

\begin{abstract}
1Département des sciences de l'activité physique, Université du Québec à Montréal, 141 Avenue du Président-Kennedy, Office SB-4390, Montreal, H2X 1Y4, Canada

2Département de chirurgie, Faculté de médecine, Université de Montréal, P.O. Box 6128, Downtown Station, Montréal, H3C 3J7, Canada

${ }^{3}$ Services de neurologie du Centre Hospitalier de l'Université de Montréal (CHUM) and Département de médecine, Université de Montréal, C.P. 6128, succursale Centre-ville, Montreal, H3C 3J7, Canada ${ }^{4}$ École de kinésiologie et des sciences de l'activité physique, Faculté de médecine, Université de Montréal, 2100 boul. Édouard-Montpetit, Montréal, H3T 1J4, Canada and Institut universitaire de gériatrie de Montréal, 4565 Chemin Queen Mary, Montréal, H3W 1W5, Canada
\end{abstract}

\section{Abstract}

We explored if adolescents with Gilles de la Tourette syndrome (GTS) had functional postural control impairments and how these deficits are linked to a disturbance in the processing and integration of sensory information. We evaluated the displacements of the center of pressure (COP) during maximal leaning in four directions (forward, backward, rightward, leftward) and under three sensory conditions (eyes open, eyes closed, eyes closed standing on foam). GTS adolescents showed deficits in postural stability and in lateral postural adjustments but they had similar maximal COP excursion than the control group. The postural performance of the GTS group was poorer in the eyes open condition (time to phase 1 onset, max-mean COP). Moreover, they displayed a poorer ability to maintain the maximum leaning position under the eyes open condition during mediolateral leaning tasks. By contrast, during forward leaning, they showed larger min-max ranges than control subjects while standing on the foam with the eyes closed. Together, these findings support the idea that GTS produces subclinical postural control deficits. Importantly, our results suggest that postural control disorders in GTS are highly sensitive to voluntary postural leaning tasks which have high demand for multimodal sensory integration.

\section{More Information}

*Address for Correspondence:

Mariève Blanchet, Professeure au département des sciences de l'activité physique de l'UQÀM,

Co-directrice du Laboratoire de recherche en motricité de l'enfant, 141 avenue du PrésidentKennedy, bureau SB-4390, 514-987-3000 poste 3742, Canada, Email: blanchet.marieve@uqam.ca

Submitted: January 06, 2021

Approved: January 29, 2021

Published: January 30, 2021

How to cite this article: Blanchet M, Prince F, Lemay M, Chouinard S, Messier J. Maximal stability limits in adolescents with Tourette syndrome. J Adv Pediatr Child Health. 2021; 4: 013-022.

\section{DOI: 10.29328/journal.japch.1001024}

Copyright: @ 2021 Blanchet M, et al. This is an open access article distributed under the Creative Commons Attribution License, which permits unrestricted use, distribution, and reproduction in any medium, provided the original work is properly cited.

D) Check for updates

OPEN ACCESS

\section{Introduction}

Gilles de la Tourette syndrome (GTS) is a neurodevelopmental disorder characterized by the presence and persistence of motor and vocal tics often (90\%) associated with various psychiatric comorbidities such as hyperactivity and obsessive-compulsive disorder [1,2]. A dysfunction of basal ganglia (BG) cortical circuitry has been shown to be involved in GTS pathophysiology [3-6].

Postural control is a fundamental aspect of daily motor acts. It requires the maintenance of the center of pressure (COP) within the base of support delimited by the feet [7]. The perception of balance control and the limits of postural stability depends crucially on the appropriate processing and integration of tactile, proprioceptive, visual and vestibular sensory signals [8,9]. BG cortical circuitry is a major contributor of the multisensory network for postural control [10]. Postural instabilities are a trademark of many BG disorders such as Parkinson's and Huntington's diseases [9,11-14]. Over the recent years, one hypothesis that has been put forward to explain postural control deficits in patients with damage in BG cortical circuits is the abnormalities in the processing and integration of sensory information particularly proprioceptive signals $[6,11,15,16]$. Several studies using reaching and grasping movement paradigms support the role of the sensory system in the pathophysiology of Tourette's syndrome [6,17-21]. Specifically, it has been shown that patients with GTS are more dependent on visual feedback when performing upper-limb movements [20]. Yet, increased reliance on visual cues has often been considered as a marker of altered proprioceptive processing [16].

Most functional movements of everyday life are performed in dynamic postural conditions requiring precise coordination between posture and voluntary movements that 
largely depend on processing, integration and reweighting of various sensory information, especially of proprioceptive signals [12]. However, only two previous studies investigated this hypothesis. In one study, the postural performance of adolescents with GTS was assessed using a quiet standing postural task performed with eyes open and eyes closed condition [22]. The results revealed postural control deficits that were independent of the visual condition. A more recent study examined postural control in GTS using altered external sensory conditions. With the Sensory Organization Test, the researchers reported greater postural instabilities in GTS, particularly when vision was removed and somatosensory cues were perturbed, leaving only the vestibular signals as a reliable source of sensory information for postural stability control [23]. To our knowledge, no previous study has investigated the dynamic postural control and the postural stability limits in patients with GTS. Thus, the hypotheses that abnormalities in the processing and integration of sensory information, particularly proprioceptive signals explain postural control deficits in patients with damage in BG cortical circuits have not been examined yet in specific GTS BG dysfunctions. The present study was important to explore these unknown symptoms. Actually, postural control in GTS was not systematically evaluated in clinical practice. The potential sensorimotor postural impairments in children with GTS could have a huge impact on their development and in their life. Indeed, it is well known that effective postural control development is associated with fundamental motor development, mobility function and social interaction [24]. Furthermore, Weisman and their colleagues [25] indicated that the presence of somatosensory dysregulation was associated with more severe impairments in quality of life and less participation in daily activities in adults with GTS. Thus, during the sensitive period of postural control development, it is critical to identify as soon as possible the children with postural control deficits for beginning specific sensorimotor stimulation [26]. These investigations are also essential to supplement the basal ganglia model with the specific basal ganglia deficits identified in the GTS. Our results could lead to a better understanding the neurophysiopathology of these structures, especially for specific sibling treatment in the pharmaceutical and the rehabilitation domain. These knowledge could also contribute to identifying the risk of falling in this pediatric population.

The present study explored, for the first time, the postural control of patients with GTS in a voluntary unperturbed maximal leaning task in four directions and three sensory conditions that increase the proprioceptive demands. This will allow us to test if postural impairments of adolescents with GTS increase when required to use a fine timevarying proprioceptive signals to control posture [27]. We hypothesized that if participants with GTS rely more on visual feedback than healthy subjects due to impaired proprioception, they should show reduced postural stability compared to healthy participants in the eyes closed conditions, especially when standing on a foam. Conversely, if GTS adolescents have a global impairment in sensorimotor integration mechanisms for postural control, then anomalies in postural control should be observed in all sensory conditions.

\section{Methods}

\section{Participants}

Twenty-four adolescents aged 10 to 14 years old were recruited to participate in this study and were divided into two groups. The first group was composed of 13 healthy adolescents $(12.2 \pm 2$ years old) with no history of motor or psychiatric disorders. The second group was composed of 13 adolescents with GTS. However, two GTS participants were excluded because they were highly hyperactive and were unable to complete the experimental task. Thus, 11 adolescents with GTS were included in the final statistical analysis (11.9 \pm 2 years old). The test $\mathrm{T}$ revealed no significant difference between groups. Participants with GTS were recruited from the Montreal Tourette Study Group in the Centre Hospitalier de l'Université de Montréal and their tics and comorbidities were evaluated by trained professionals. GTS was diagnosed using criteria from the Tourette Syndrome Classification Study Group [28]. Every GTS participant in this study met all the evaluation criteria of this evaluation and they were put in the definite category. Obsessive-compulsive disorder (OCD) symptoms were evaluated using the YaleBrown Obsessive-Compulsive Scale (Y-BOCS) [29] and attention deficit-hyperactivity disorder (ADHD) symptoms were evaluated through an interview using DSM-IV criteria and hyperactivity-impulsivity criteria. The comorbidities and medications are presented in table 1.

\begin{tabular}{|c|c|c|c|c|}
\hline Participants & Medication & $\begin{array}{c}\text { Medication } \\
\text { during testing }\end{array}$ & ADHD & OCD \\
\hline GTS1 & No & no & no & $\begin{array}{c}\text { OCD } \\
\text { definite }\end{array}$ \\
\hline GTS2 & No & no & combined type & $\begin{array}{c}\text { OCD } \\
\text { probable }\end{array}$ \\
\hline GTS3 & Concerta & no & no & $\begin{array}{c}\text { OCD } \\
\text { probable }\end{array}$ \\
\hline GTS4 & $\begin{array}{l}\text { Risperidone, } \\
\text { Clonazepam }\end{array}$ & yes & inattentive type & $\begin{array}{c}\text { OCD } \\
\text { probable }\end{array}$ \\
\hline GTS5 & No & no & inattentive type & no \\
\hline GTS6 & Concerta & no & combined type & no \\
\hline GTS7 & No & no & inattentive type & no \\
\hline GTS8 & $\begin{array}{c}\text { Concerta, } \\
\text { Ritalin, Adderal }\end{array}$ & no & inattentive type & $\begin{array}{c}\text { OCD } \\
\text { definite }\end{array}$ \\
\hline GTS9 & $\begin{array}{l}\text { Strattera, } \\
\text { Concerta }\end{array}$ & $\begin{array}{l}\text { yes, only } \\
\text { Strattera }\end{array}$ & $\begin{array}{c}\text { hyperactive-impulsive } \\
\text { type }\end{array}$ & $\begin{array}{c}\text { OCD } \\
\text { definite }\end{array}$ \\
\hline GTS10 & Riva-citalopram & yes & no & $\begin{array}{c}\text { OCD } \\
\text { definite }\end{array}$ \\
\hline GTS11 & $\begin{array}{l}\text { Strattera, } \\
\text { Concerta }\end{array}$ & $\begin{array}{l}\text { yes, only } \\
\text { Strattera }\end{array}$ & combined type & no \\
\hline
\end{tabular}


The project has been approved by the CERSS of the University of Montreal, CERSS-1005. All participants and their parents have given their written consent to participate.

We verified whether the effects observed on the entire sample were due to a subgroup with a specific medication. If the participant were taking any kind of neuroleptics, clonidine, or stimulants he was excluded from the analysis. Analyses revealed that the postural group differences were still present [22]. We also verified if the postural performance was correlated with the medication, with the comorbidities and with the tics severity, but no significant value was observed. In the light of our sample, the statistical analyses indicated that this study is a third to demonstrate that these three factors have no significant effect of the postural control deficits observed in GTS [22].

In order to reduce the adolescent's stress, the experimenter took 10 minutes with each participant in both groups to create a social relation and often asked if they had questions during the explanations of the tasks. The experimenter said, "There is no right answer for the task execution, you just have to maintain your maximum leaning position." $\mathrm{He}$ also mentioned at the adolescents with the GTS: "feel free to let your tics come and go before and/or after the maximum leaning tasks. Moreover, in order to make sure that motor or sensory tics did not interfere with the task: 1) we rejected the trial where a motor or vocal tic was present, 2) participants were asked if a sensory tic was felt during the trial, 3) all trials were visually inspected during the experimentation and after with the COP displacements. All the participants had executed the tasks without observables or self-reported tics.

The study was approved by the university ethics committee and all parents and participants provided written informed consent or assent.

\section{Experimental setup and procedures}

The experimental tasks assessed postural control abilities during maintenance leaning period. At the beginning of the trial, participants stood barefoot on a force plate with their arms crossed on their chest. They were asked to maintain upright quiet standing position until an auditory cue (5 s) instructed the participants to lean as far as possible in different directions for each trial (forward, backward, rightward and leftward) and to maintain this maximum leaning position. After $10 \mathrm{~s}$, a second auditory cue instructed the participant to return to the initial quiet standing position for $7 \mathrm{~s}$ (total $22 \mathrm{~s}$ ). During the experimental task, participants were asked to keep their body straight without moving or lifting their feet or flexing their hips or knees $[9,13,27]$. These experimental tasks were tested in three different sensory conditions: a) eyes open while standing on a firm surface (EO), b) eyes closed while standing on a firm surface (EC on a firm) and c) eyes closed while standing on a foam (EC on foam). The compliant foot support was a $5.5 \mathrm{~cm}$-thick medium density foam. In EO condition, participants were asked to fix a target ( $2 \mathrm{~cm}$ of diameter) on the wall 3 meters away from them. During EC on a firm and EC on foam conditions, participants were encouraged to remember the target's position and were asked to open their eyes between each trial in order to keep their head position in approximately the same position across trials (for example, avoid bending the head). The investigator also observed the head orientation and the respect of general postural instructions before and during the total duration of the task. In the EO condition, we tested the postural abilities of participants to maintain maximum leaning position while all sensory information is available (visual, proprioceptive, tactile and vestibular). The comparison between the eyes open and eyes closed on firm conditions allowed to test whether GTS participants rely less on vision than control adolescents for dynamic postural control. The EC on foam condition was used both to decrease plantar pressure sensations as well as increase instability $[13,30]$. This allowed to evaluate if postural control of GTS participants were poorer than control group when they are force to use fine time varying proprioceptive signals to control posture. Participants had to repeat the task once time in each direction and in each sensory condition, starting by forward, backward, rightward and finished with the leftward leaning directions. A practice trial was performed before. All the participants were evaluated with the same instructor. Further details regarding methodological features are presented in Blanchet, Prince, Chouinard, \& Messier, 2014 and in Blanchet, Prince and Messier 2019 [13,27].

\section{Data collection and data analysis}

The ground reaction force data were collected at $200 \mathrm{~Hz}$ using an AMTI force plate (AMTI, model OR6-5; Advanced Mechanical Technology, Inc, Watertown, MA, USA) and data processing was performed using the Balance Clinic software. The raw data were extracted and computed by Excel (office suite) and MatLab software. The force plate was calibrated before the evaluation of each participant. The COP displacements were analyzed during the maximum leaning and were subdivided into two phases (Figure 1). The beginning of phase 1 was defined as the first time the COP moved in the opposite direction to the voluntary leaning movement (namely Time to phase 1 onset) and ended at the $5^{\text {th }}$ second of maintain their maximum leaning. The approximate required time to phase 1 onset was $1.5 \mathrm{~s}$ in means (standard deviation $0.46 \mathrm{~s}$ ) but this time was affected by sensory condition and by the GTS (see results section). The phase 1 reflected the initial stabilization period affected by the momentum of the voluntary leaning movement and the strategy used to reach the maximum leaning. Phase 2 was during the last $5 \mathrm{~s}$ of maintaining their maximum leaning. Both phases allowed to assess postural control over time i.e., during the initial postural adjustments after reaching the stability limits and during the maintenance of the maximum leaning position. 
In both phases, COP displacements were analyzed. First, the min-max ranges were calculated as the distance between the maximal and the minimal COP displacement values (namely min-max range) [22,31] along the axis of leaning movement (anteroposterior (AP) during forward and backward leaning and mediolateral (ML) axes during rightward and leftward leaning) as well as along the opposite axis of leaning movement (AP during rightward and leftward leaning and ML axes during forward and backward leaning). The analyses of the amplitude of the COP along the opposite axis during the voluntary movement are reported to illustrate lateral postural adjustments or asymmetries [9]. For these variables, the larger values indicated a poorer postural performance. These dependent variables revealed the maximum amplitude of COP displacements (stability indication) but does not provide information on the ability to maintain their COP near the stability limits. Therefore, another original dependent variable was created to reflect this important postural control skill. We calculated the distance between the maximal COP value and the mean position of COP displacements (COPmax-COPmean). The smaller values revealed a better postural performance [13]. Finally, we had calculated the maximum excursion of the COP from quiet standing to maximum lean. This variable, named maximum COP excursion, is largely reported in stability limits studies $[9,32]$.

\section{Statistical analysis}

Before starting the analysis, all results were normally distributed. To determine whether sensory conditions and leaning movement directions influenced postural stability during maximum leaning in both SGT and control adolescents, a repeated-measure ANOVA with a between factor (group) and 2 within factors (direction and sensory condition) was applied on the amplitude (COP ranges and COPmax-COPmean) values of COP displacements and Time to phase 1 onset. These analyses were performed in both phases for the leaning period. Greenhouse-Geisser corrections were applied to all ANOVAs to reduce the probability of type I error. Also, Post hoc pairwise comparisons were made using Bonferroni correction. For conciseness, only main effects and interactions including the factor group are reported for all analyses of variance.

\section{Results}

\section{Time to phase 1 onset}

The ANOVAs conducted on the Time to phase 1 onset variable indicated a significant interaction effect between groups and conditions interaction $(\mathrm{F}(1,22)=4.047, p<0.05)$. The Post-hoc analyses revealed that adolescents with GTS required significantly more time to reach phase 1 in EO condition than healthy adolescents $(p<0.05)$.

\section{Min-max range}

During the first phase of maximum leaning, the ANOVAs showed a significant main effect of group indicating that the min-max range of adolescents with GTS were larger than those of healthy controls when computed along the axis of

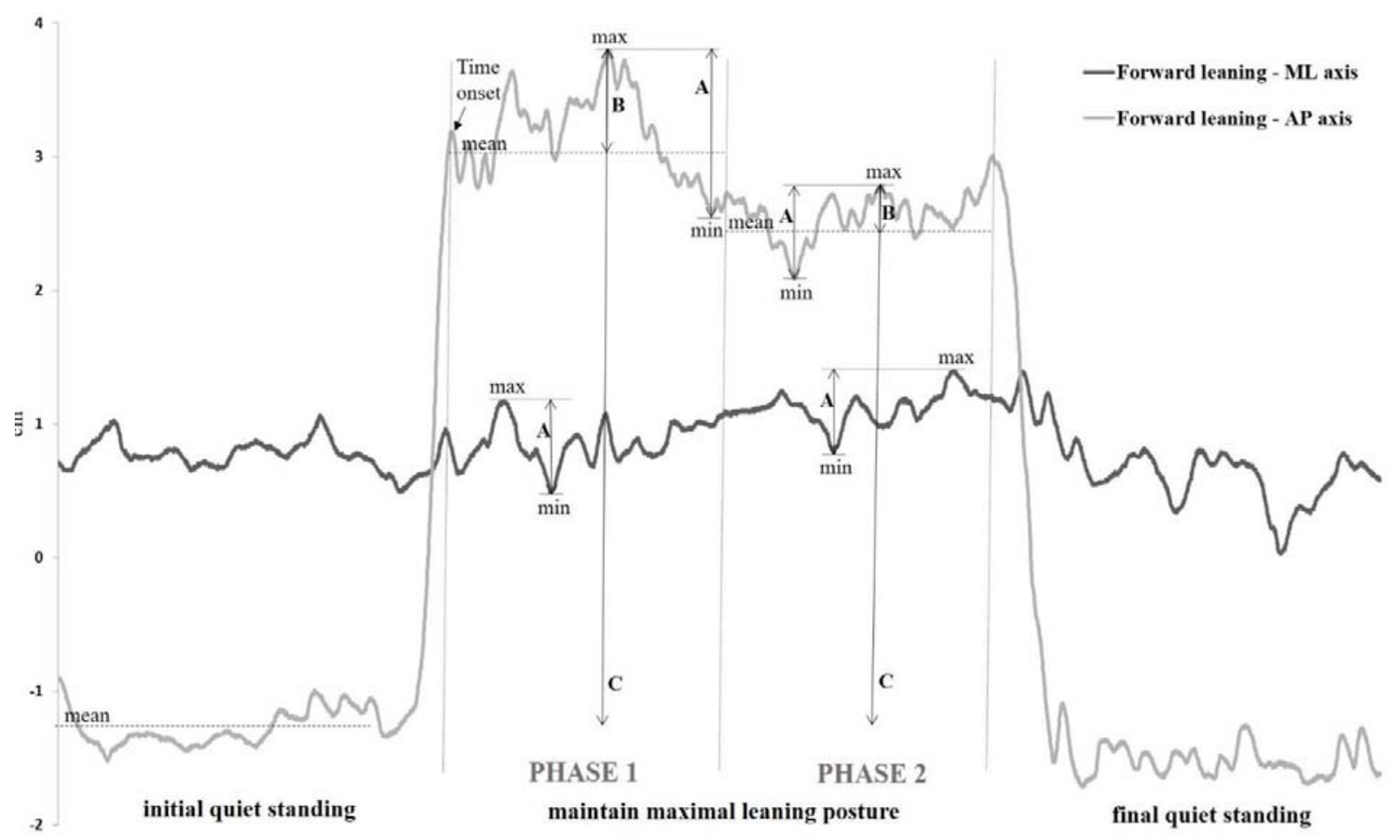

Figure 1: Parameters considered in the data analysis during both phases are shown from a representative adolescent with GTS. The light gray line represents the AP COP excursion during a complete trial in the forward direction and the dark gray line represents the one in ML axis. The A represent min-max ranges, the $B$ represent COPmax-COPmean and the $C$ represent the maximum excursion of the COP. 
leaning movement $(\mathrm{F}(1,22)=5.77, p<0.05)$ as well as along the opposite axis of leaning $(\mathrm{F}(1,22)=11.04, p<0.005)$. Furthermore, the analysis of min-max range computed along the axis of leaning movement indicated a significant interaction effect between groups, conditions and directions $(\mathrm{F}(6,132)=2.57, p<0.05)$ (Figure 2). Post-hoc analyses revealed that the min-max range of adolescents with GTS were larger than those of healthy controls during forward leaning in the foam condition $(p<0.05)$ as well as during rightward $(p<0.05)$ and leftward leaning $(p<0.05)$ in the eyes open condition.

During the second phase of maximum leaning, adolescents with GTS also exhibited larger min-max range values than healthy adolescents both along the leaning axis $(F(1,22)=6.43$, $p<0.05$ ) and along the axis opposite to the leaning movement $(\mathrm{F}(1,22)=7.35, p<0.05)$. However, in contrast to the first maximal leaning phase, there was no significant groups by conditions by directions interaction indicating that the difference between healthy and GTS adolescents was similar across sensory conditions and leaning movement directions.

\section{COPmax-COPmean}

Adolescents with GTS exhibited a greater overall mean level of COPmax-COPmean across sensory conditions and leaning movement directions (Figure 3). Accordingly, the
ANOVAs revealed a significant difference between groups in the magnitude of COPmax-COPmean for phase $1(\mathrm{~F}(1,22)$ $=6.69, p<0.05))$ and phase $2(\mathrm{~F}(1,22)=6.97, p<0.05))$ (Figure 3). Furthermore, the analysis indicated a significant group by condition interaction $(\mathrm{F}(2,44)=4.05, p<0.05)$ for phase 1. Post-hoc analyses revealed that adolescents with GTS displayed larger COPmax-COPmean than healthy adolescents in the eyes open condition $(p<0.05)$.

\section{Maximum COP excursions}

The ANOVAs performed on the maximum COP excursions revealed no significant group effect (phase 1 : $(\mathrm{F}(1,22)=$ $1.316, p>0.05)$; phase $2:(\mathrm{F}(1,22)=0.27, p>0.05))$, nor than interaction effect.

\section{Discussion}

For the first time, we investigated dynamic postural control and stability limits in three different sensory conditions in GTS. Participants performed a voluntary unperturbed maximal leaning task in four directions. There were three main findings in this study. First, adolescents with GTS showed postural stability impairments (phase 2) and deficits in lateral postural adjustments (both phases). Second, GTS adolescents displayed the greatest postural instabilities relative to healthy controls in the eyes open

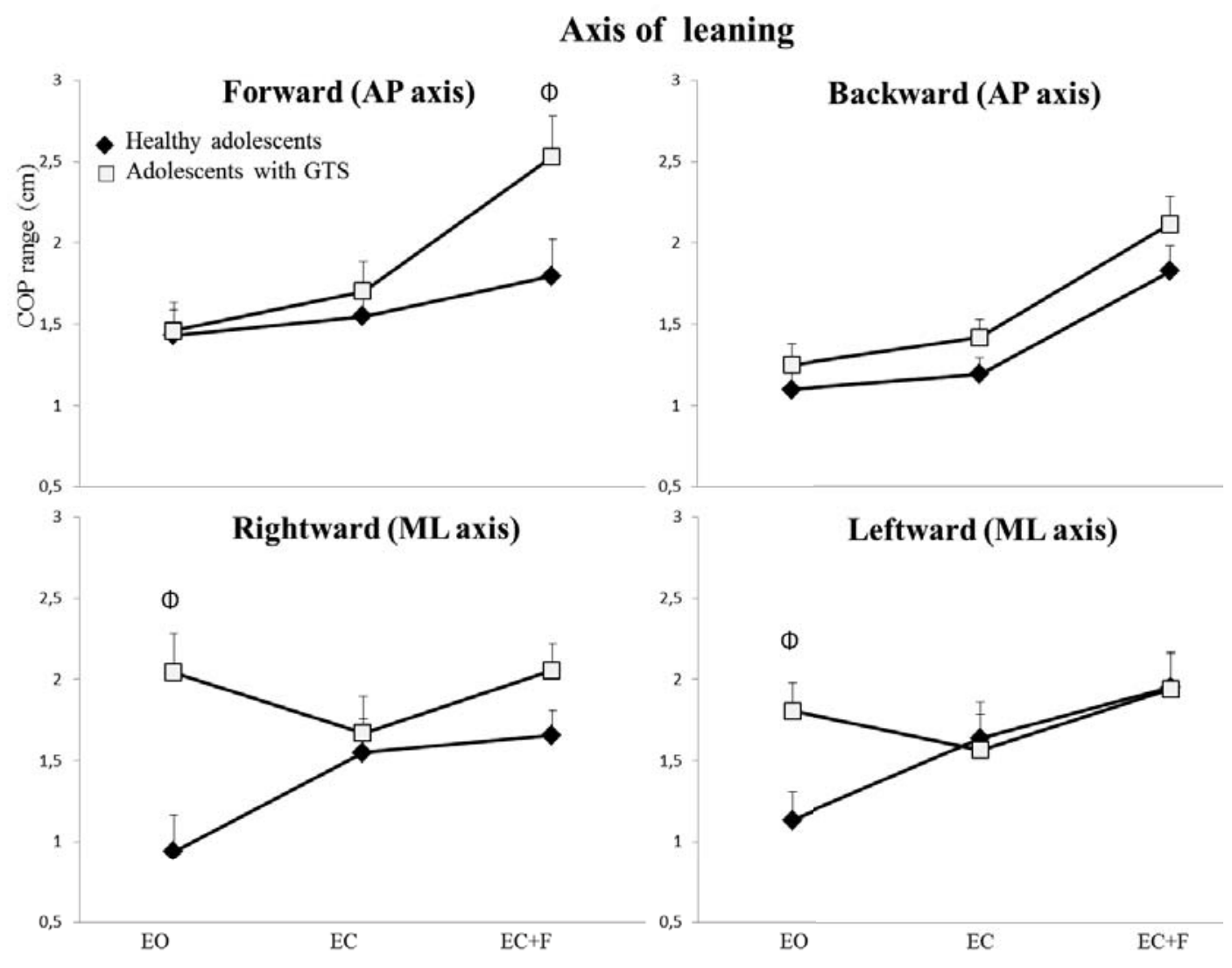

Figure 2: The $\Phi$ indicates interaction effect between the condition, the direction and the group for COP range in four directions under three sensory conditions during phase 1 for both subject's groups. Error bars represent the standard error of the mean. 

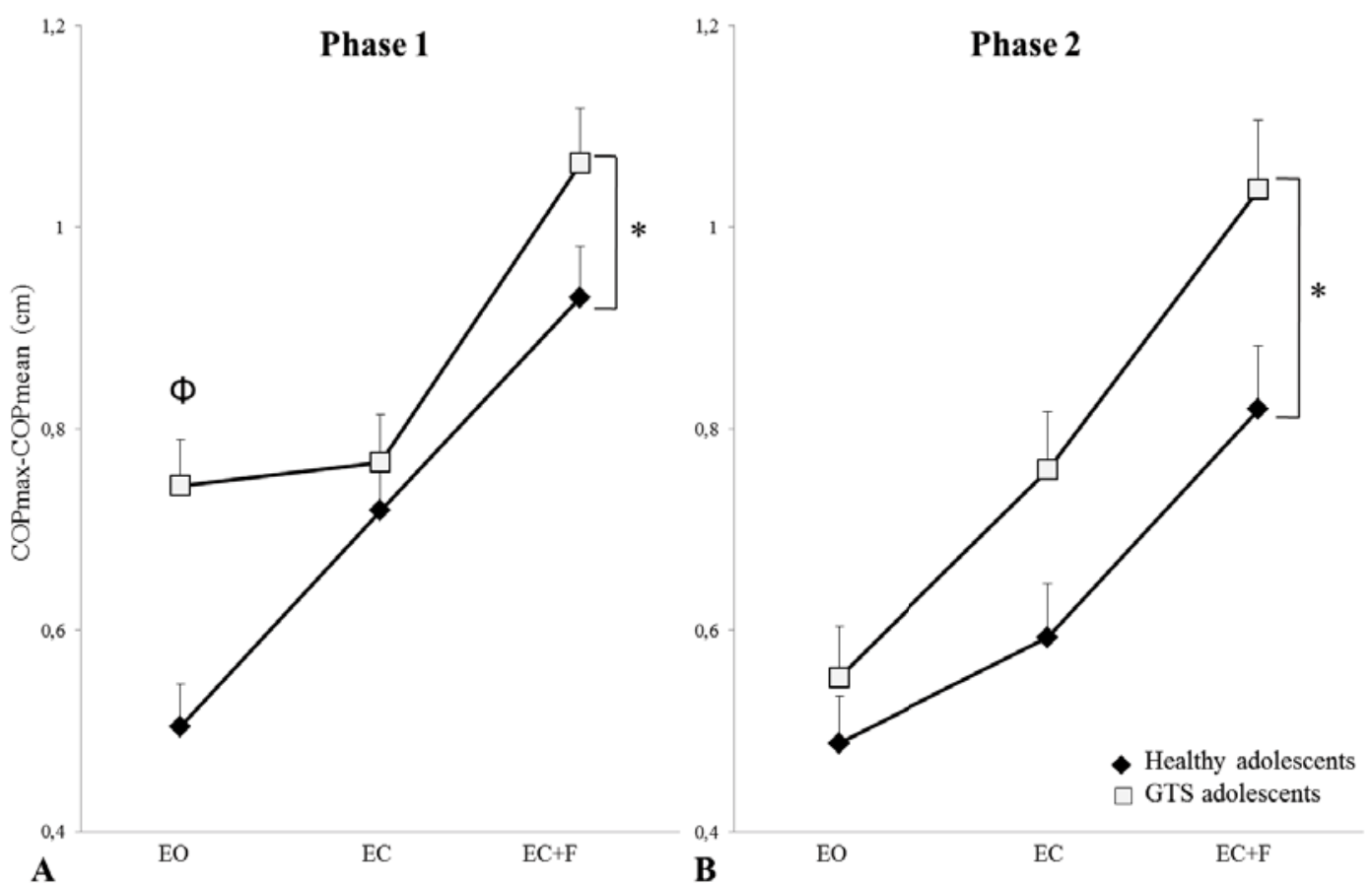

Figure 3: Independently from forward, backward, left, or right leans, the $\Phi$ reveals the significant interaction between the condition and the group for COPmax-COPmean value during the phase $1(A)$ and phase $2(B)$ under each sensory condition for both groups. Error bars represent the standard error of the mean.

and in the foam conditions during the first leaning phase. Finally, the GTS did not affect the maximal COP excursion performance. These findings are discussed in the following sections.

\section{Postural control at the limits of stability in GTS adolescents}

The present study provides evidence that GTS adolescents have subclinical dynamic postural control impairments i.e. deficits not investigated by the classical clinical neurological assessments. In the phase 2 , they exhibited larger min-max ranges along the axis of the leaning movement as well as a reduced ability to maintain the maximal inclined posture. These results are consistent with a previous study reporting higher COP ranges during quiet standing and one-legged standing in GTS participants [22].

Furthermore, the results revealed that the GTS adolescents had deficits in lateral postural adjustments or asymmetries in both phases (min-max COP along the opposite axis of leaning). Our results also agree with many other studies demonstrating postural control deficits and lateral postural adjustments or asymmetries in basal ganglia disorders, such as Parkinson's and Huntington's diseases [9,11-14]. Moreover, the postural impairments reported here are in line with the results of our previous study indicating that Huntington's disease patients show a degraded ability to maintain the maximum leaning position and lateral postural adjustments even before the clinical disease onset [13].

These results could also be explained in part by trunk dystonic postures. In fact, in 2019 , a study revealed that one of the most frequent manifestations in GTS dystonic tics is the trunk dystonic postures (59.5\%). Despite the fact that the experimenter did not observed tic manifestations during the experimental postural task, as mentioned in the methodology section, it could be possible that this type of tics could influence postural control and cause postural asymmetries.

These findings support the idea that damage in basal ganglia-cortical network produces impairments in several aspects of balance control, including the ability to coordinate postural adjustments with voluntary movements [15]. Furthermore, the current results indicate that our experimental task is sensitive to highlight balance difficulties in GTS. This is important since postural control at the limit of stability simulates functional positions occurring in daily life and is a key determinant of fall risk [9,33]. The results suggested that GTS adolescents could be more at risk of falling. Indeed, GTS adolescents reached similar maximal COP excursion than the control group but they indicated significant impairments in maintaining their balance.

\section{Sensory processing and integration in GTS adolescents}

Over the recent years, one important hypothesis that has been put forward to explain postural control deficits in basal ganglia damage patients is abnormalities in the processing and integration of sensory information, particularly proprioceptive signals $[6,15,16]$. Based on these prior works, we hypothesized that adolescents with GTS rely more on visual feedback than healthy subjects due to 
impaired proprioception. Therfore, they should show reduced postural stability compared to healthy participants in the eyes closed conditions, especially when standing on a foam. The min-max range analysis (along the axis of leaning movement) revealed poorer performance in the EC on foam condition for GTS group during the forward leaning of the first phase and support this hypothesis. In striking contrast, adolescents with GTS exhibited the greatest postural control difficulties relative to healthy participants under EO condition. Indeed, deficits showed in EO condition was observed with min-max range (along the axis of leaning movement) in leftward and rightward leaning. Moreover, when the GTS group reach their maximum leaning position, the Time to phase 1 onset (i.e. the initial postural adjustments revealed by first time the COP moved in the opposite direction to the voluntary leaning movement (Figure 1), was significantly longer in EO condition (0.5 s) despite the fact that they reach similar maximal COP excursions than the control group. Their capacities to maintain maximal leaning position (COPmax-COPmeans) also were more affected by EO condition. The EO and EC on foam conditions might have been especially challenging for GTS participants for different reasons.

First, the greater impairments of GTS adolescents in the EO condition might be linked to specific difficulties in visual sensory integration for dynamic postural control. Some previous studies reported deficient visual processing in GTS participants. For instance, two studies revealed visuomotor integration alterations in fine motor skills of the upper limb using the standardized pegboard of the Motorische Leistungsserie system [21] and the Berry visual-motor integration test [17]. However, in static postural control, Lemay and their colleagues [22] reported no statistical difference between children with GTS and their pairs in EO and EC on a firm surface conditions [22]. The motor control requirements in static balance paradigm used in their study compared to the one employed in our experimental design task could explain in part, these observations. Interestingly, the initial postural stabilization was highly sensitive to the sensory processing and integration impairments for dynamic postural control in GTS. The interaction effects between the group and the sensory conditions were only exhibited in the phase 1 . No interaction was demonstrated in the phase 2 . These observations suggest that initial stabilization period and late phases of maintaining maximal leaning position involve different balance constraints having differential demands for sensorimotor integration. Indeed, compared to classical static stability assessments or unpredictable short postural perturbations that largely involved subcortical pathways, brainstem and spinal structures [34], the voluntary unperturbed self-paced postural movements favor the recruitment of cortical activities. In this light, the network used to achieve the voluntary leaning tasks in the phase 1 that soliciting both the feed forward (planning) and feedback (online) process could highlight specific sensory disturbances for dynamic postural control in GTS that did not appear in quiet standing task [22]. In contrast to the phase 1 , the phase 2 possibly solicited online mechanisms who gets closer to the one used in static postural control. Indeed, holding the COP near the limits of foot support in the phase 2 could be less dependent on the planning process and/or of sensory information and could explain the different results observed in both phases. This hypothesis is supported by our results obtained in phase 2 which are similar to the results demonstrated in a quiet standing [22].

The initial postural instabilities of GTS adolescents may also be linked to the use of different strategies than control participants to reach their limits of stability. A previous study exploring postural stability limits in Parkinson's disease has reported the use of a different spatial-temporal pattern during the motion phase of the learning task [9]. Other studies have likewise reported a breakdown in the temporal coordination between postural adjustments and voluntary movements in Parkinsonian patients [12]. However, these studies did not use various sensory conditions in their experimental protocol. The impairments in the Time to phase 1 onset and in their capacities to maintain maximal leaning position (COPmaxCOPmeans) support that the temporal breakdown between the voluntary leaning movement and postural adjustments may be responsible for the marked postural instabilities measured during the initial leaning phase of GTS adolescents in EO.

Since all sensory modalities are available for postural control in the EO (vision, proprioceptive, tactile and vestibular), postural dysfunctions in this condition might also reflect a sensory treatment overload. Indeed, it has been hypothesized that the symptoms characterizing GTS occur as a result of the failure of sensory gating, a concept which describes the prevention of sensory overload by filtering out irrelevant stimuli $[4,19,35,36]$.

The EC on foam condition also involved complex sensorimotor mechanisms, especially in terms of proprioceptive processing. The foam condition alters the plantar pressure sensations compared to standing on a firm surface and increases instability at the whole body. Thus, without vision participants must use a fine time varying proprioceptive signals to control posture. In patients with Parkinson's and Huntington's disease, postural control anomalies have been attributed to a disturbance in proprioception [13,37]. Although no previous study has directly assessed proprioceptive sensitivity in GTS adolescents, altered perception of internal sensations has been repetitively reported in GTS [38] and the quantitative sensory testing revealed that $15 \%$ of adults with GTS had suspected somatosensory dysregulation and 35\% had somatosensory dysregulation [25]. These observations might also explain the greater deficits of GTS adolescents in the EC on foam condition. 
However, it has been suggested that when proprioception is highly challenged, as it is the case when standing on foam support surface without vision cues, subjects tended to rely also on vestibular signals for postural control [8]. One recent report indicated that GTS children are particularly impaired in controlling posture when vestibular information was challenged [23]. However, it is worth mentioning that, in this previous study researcher assessed postural control under a perturbation paradigm where proprioceptive sensations were made inaccurate, thereby forcing children to rely on vestibular sensations. By contrast, in the current study, proprioceptive processing was challenged using a voluntary unperturbed leaning task. In our foam condition, proprioceptive cues are markedly different from standing on a firm surface, as it is the case when standing on an exercise mat or on sand. Notably, however, the proprioceptive signals are entirely reliable and representative of the postural context. Therefore, we believe that subjects needed to use proprioceptive cues to produce fine context adapted postural adjustments. Our paradigm does not allow dissociation between these alternative hypotheses. However, since a plethora of studies reported impaired proprioception in $B G$ disorders and the processing of proprioceptive information is especially complex in our foam condition, we believe that the observed greater stability difficulties (min-max range) of GTS adolescents during forward leaning in the EC on foam condition largely reflects altered proprioceptive processing mechanisms.

Altogether, these hypotheses could not, however, explain why the GTS adolescents exhibited different sensory symptoms in AP and in ML leaning axes in phase 1. It is well known that the central nervous system may use different postural strategies to control the AP versus ML components of postural stability [7]. Our study could bring new insight about the different sensory weighting required to control AP versus ML components of postural stability. In other words, the relative contribution of each sensory modality to control the AP versus ML components was possibly different. The results might indicate that, in GTS adolescents, the AP postural control is more dependent on proprioceptive information and the one in ML may be more dependent on visual information. In anterior postural leaning, the task depends largely on the precise activity of the antigravity muscles that are greatly involved in the control of balance since a very young age. It is possible that the forward leaning task depends more on the fine time-varying proprioceptive signals. To our knowledge, no previous study had evaluated this interesting hypothesis but deserve to be deepened.

Another possible explanation of the deficits observed in GTS in phase 2 is a deficit in sensorimotor integration mechanisms. Multiple lines of evidence indicate that the basal ganglia are a major contributor to sensorimotor integration mechanisms [16]. One previous study has suggested that such real-time control of quiet postural stability might be impaired in GTS [22]. Other studies have likewise suggested that BG disorders led to impair online movement control [39]. Additionally, some previous studies have suggested that Parkinson's disease patients [16,40] and GTS children [22] have intermodal sensorimotor integration deficiencies. Hence, several reports indicated that normal postural development involves both a more effective use of sensory information as well as better sensorimotor integration [31,41]. It is possible that the neurodevelopmental disorders of GTS increased the delay in the development of these mechanisms and explain the postural disorders in phase 2 .

Altogether, the results of this study highlighted the importance of the clinical sensorimotor postural control assessments in GTS's patients. This study also indicated that the functional reach test was probably not the most appropriated postural test because the maximal COP excursion revealed no significant difference. The clinical assessments with pediatric balance measures must, however, include various components of balance including static and dynamic stability, planning treatment programs and sensory integration.

\section{Other disease-related considerations}

Other GTS neurological changes could also be contributing to the sensorimotor disturbances observed in this study. Firstly, several structural abnormalities have been observed in the parietal cortex, thalamus, somatosensory cortex, corpus callosum, premotor cortex of individuals with GTS [42-46]. Moreover, studies have reported increased activity in the somatosensory cortex, supplementary motor cortex and primary motor cortex before and during voluntary upper limb movements [19,47]. Thus, in addition to the dysfunctions in basal ganglia network, several neural abnormalities could also be responsible for the sensorimotor deficits in GTS. However, it is important to note that the majority of these neurophysiological results were obtained using voluntary distal upper limb motor tasks. This type of task primarily involves the cortico-striato-thalamo-cortical networks [35]. In contrast, unperturbed dynamic postural control involves more largely the cortico-basal ganglia-brainstem networks. Thus, only a very tentative correlation can be made between our results and the observations reported in anterior studies using upper limb motor tasks in GTS.

\section{Limits of the study}

In this exploratory study, the COP analysis itself could not permit confirming the postural strategy hypothesis postulated in the phase 1. Future studies involving both COP displacements and kinematic analyses are needed to better assess how GTS impacts on the coupling between voluntary movements and postural adjustments in different sensory conditions. The small sample of participants could not allow to generalize the interpretation of the results. This study was, 
however, required for preliminary investigations of these important hypotheses. The limited number of trials in each direction and each condition could affect the reliability of the data. This protocol selection was based on our previous studies and our pilot studies. The experimental protocol was a compromise between minimize the fatigue effect and have 3 sensory conditions as well as 4 directions to evaluate our hypotheses in a pediatric population with neurological disorders. Finally, the anomalies observed in the postural control of GTS adolescents might also be linked to various aspects of the tics and/or comorbidities. It could be possible that the motor, sensory, premonitory urge, OCD and/or ADHD had affected our postural data.

\section{Conclusion}

The clinical expression of GTS is highly heterogeneous. Our findings demonstrated subclinical deficits in dynamic postural control in adolescents with GTS, especially in the phase 2 . They also demonstrated lateral postural adjustments in both phases. The postural abilities in the leaning position was more greatly impaired by the sensory conditions in the phase 1 . This study raises the hypothesis that postural disturbances are more accentuated when GTS participants have high demand for multisensory signals during the initial stabilization of leaning position such as revealed by the time to phase 1 onset, the capacities to maintain the maximal inclined posture and the min-max range in ML leaning directions. In contrast, the proprioceptive processing was significantly altered during forward leaning (min-max range). These results could reveal new insight about the different sensory weighting required to control AP versus ML components of postural stability. However, these hypotheses need further investigations. Further investigations involving various dynamic or functional postural paradigms and larger participants groups are required to elucidate how impairments in specific sensory systems contribute to postural control difficulties in GTS. With future longitudinal studies, in a larger population, deficits in this dynamic postural task may become a useful biomarker to characterize anomalies in postural control development and to identify an endophenotype in GTS. Furthermore, these postural analyses might serve as outcome measures to objectively assess the effectiveness of specific balance training in GTS.

\section{Acknowledgement}

This work was supported by the GO Foundation in Quebec and by the CHU Sainte-Justine hospital (Fondation des étoiles) Québec-Canada. We wish to thank the Montreal Tourette study group, Raphaël Hart, Catherine Lefrançois and Francine Pilon. We also wish to thank Marcel Beaulieu (computer engineering) for his expert technical assistance.

\section{References}

1. Eddy $\mathrm{CM}$, Cavanna $\mathrm{AE}$, Gulisano $\mathrm{M}$, Calì $\mathrm{P}$, Robertson $\mathrm{MM}$, et al The effects of comorbid obsessive-compulsive disorder and attention- deficit hyperactivity disorder on quality of life in tourette syndrome. $\mathrm{J}$ Neuropsychiatry Clin Neurosci Fall. 2012; 24: 458-462.

PubMed: https://pubmed.ncbi.nlm.nih.gov/23224452/

2. Hirschtritt ME, Lee PC, Pauls DL, Dion Y, Grados MA, et al. Tourette Syndrome Association International Consortium for Genetics. Lifetime prevalence, age of risk, and genetic relationships of comorbid psychiatric disorders in Tourette syndrome. JAMA Psychiatry 2015; 72: 325-333. PubMed: https://pubmed.ncbi.nlm.nih.gov/25671412/

3. Mink JW. Basal ganglia dysfunction in Tourette's syndrome: A New Hypothesis. Pediatr Neurol. 2001; 25: 190-198.

PubMed: https://pubmed.ncbi.nlm.nih.gov/11587872/

4. Leckman JF, Bloch MH, Smith ME, Larabi D, Hampson M, et al Neurobiological substrates of Tourette's disorder. J Child Adolesc Psychopharmacol. 2010; 20: 237-247.

PubMed: https://www.ncbi.nlm.nih.gov/pmc/articles/PMC2958453/

5. Worbe $Y$, Malherbe C, Hartmann A, Pélégrini-Issac M, Messé A, et al Functional immaturity of cortico-basal ganglia networks in Gilles de la Tourette syndrome. Brain. 2012; 135:1937-1946.

PubMed: https://pubmed.ncbi.nlm.nih.gov/22434213/

6. Patel N, Jankovic J, Hallett M. Sensory aspects of movement disorders. Lancet Neurol. 2014; 13: 100-112.

PubMed: https://pubmed.ncbi.nlm.nih.gov/24331796/

7. Winter DA, Prince F, Frank JS, Powell C, Zabjek KF, et al. Unified theory regarding $A / P$ and $M / L$ balance in quiet stance. J Neurophysiol. 1996; 75: 2334-2343.

PubMed: https://pubmed.ncbi.nlm.nih.gov/8793746/

8. Peterka RJ. Sensorimotor integration in human postural control, J Neurophysiol. 2002; 88: 1097-1118.

PubMed: https://pubmed.ncbi.nlm.nih.gov/12205132/

9. Mancini M, Rocchi L, Horak FB, Chiari L. Effects of Parkinson's disease and levodopa on functional limits of stability. Clin Biomechanics. 2008; 23: $450-458$

10. Nagy A, Eördegh G, Paróczy Z, Márkus Z, Benedek G, et al Multisensory integration in the basal ganglia. Eur $\mathrm{J}$ Neuroscience. 2006; 24: 917-924.

PubMed: https://pubmed.ncbi.nlm.nih.gov/16930419/

11. Abbruzzese G, Berardelli A. Sensorimotor integration in movement disorders. Mov Disord. 2003; 18: 231-240.

PubMed: https://pubmed.ncbi.nlm.nih.gov/12621626/

12. Tagliabue M, Ferrigno G, Horak F. Effects of Parkinson's disease on proprioceptive control of posture and reaching while standing. Neuroscience. 2009; 158: 1206-1214.

PubMed: https://pubmed.ncbi.nlm.nih.gov/19136043/

13. Blanchet M, Prince F, Chouinard S, Messier J, et al. Postural stability limits in manifest and premanifest Huntington's disease under different sensory conditions. Neuroscience. 2014; 279: 102-112.

PubMed: https://pubmed.ncbi.nlm.nih.gov/25168735/

14. Tian JR, Herdman SJ, Zee DS, Folstein SE, et al. Postural control in Huntington's disease, Acta Oto-Laryngologica. Supplementum. 1991; 481: 333-336.

PubMed: https://pubmed.ncbi.nlm.nih.gov/1833947/

15. Visser JE, Bloem BR. Role of the basal ganglia in balance control. Neural Plasticity. 2005; 12: 161-174.

PubMed: https://pubmed.ncbi.nlm.nih.gov/16097484/

16. Konczak J, Corcos DM, Horak F, Poizner H, Shapiro M, et al. Proprioception and motor control in Parkinson's disease. J Motor Behav. 2009; 41: 543-552.

PubMed: https://pubmed.ncbi.nlm.nih.gov/19592360/

17. Schultz RT, Carter AS, Gladstone M, Scahill L, Leckman JF, et al. Visual-motor integration functioning in children with Tourette syndrome. Neuropsychology. 1998; 12: 134-145.

PubMed: https://pubmed.ncbi.nlm.nih.gov/9460741/ 
18. BerardelliA, CurràA, FabbriniG, GilioF,ManfrediM, etal.Pathophysiology of tics and Tourette syndrome. J Neurol. 2003; 250: 781-787. PubMed: https://pubmed.ncbi.nlm.nih.gov/12883917/

19. Biermann-Ruben K, Miller A, Franzkowiak S, Finis J, Pollok B, et al. Increased sensory feedback in Tourette syndrome. Neurolmage. 2012; 63: $119-125$

PubMed: https://pubmed.ncbi.nlm.nih.gov/22776453/

20. Georgiou N, Bradshaw JL, Phillips JG, Bradshaw JA, Chiu E, et al Advance information and movement sequencing in Gilles de la Tourette's syndrome. J Neurol Neurosurg Psychiatry. 1995; 58: 184-191. PubMed: https://www.ncbi.nlm.nih.gov/pmc/articles/PMC1073315/

21. Neuner I, Arrubla J, Ehlen C, Janouschek H, Nordt C, et al. Fine motor skills in adult Tourette patients are task-dependent, BMC Neurol. 2012; 11: 120-128.

PubMed: https://pubmed.ncbi.nlm.nih.gov/23057645/

22. Lemay M, Termoz N, Lesperance P, Chouinard S, Rouleau GA, et al. Postural control anomalies in children with Tourette syndrome, Experimental Brain Res. 2007; 179: 525-530.

PubMed: https://pubmed.ncbi.nlm.nih.gov/17342479/

23. Liu WY, Ya-Ting H, Lien HY, Wang HS, Wong AM, et al. Deficits in sensory organization for postural stability in children with Tourette syndrome. Clin Neurol Neurosurg. 2015; 129: S36-40.

PubMed: https://pubmed.ncbi.nlm.nih.gov/25683311/

24. Assaiante C. Action and representation of action during childhood and adolescence: A functional approach. Neurophysiologie Clinique/ Clinical Neurophysiology. 2011; 42: 43-51.

PubMed: https://pubmed.ncbi.nlm.nih.gov/22200341/

25. Weisman H, Parush S, Apter A, Fennig S, Benaroya-Milshtein N, et al. A study of sensory dysregulation in children with tic disorders. J Neural Transm. 2018; 125: 1077-1085.

PubMed: https://pubmed.ncbi.nlm.nih.gov/29473112/

26. Ismail FY, Fatemi A, Johnston MV. Cerebral plasticity: windows of opportunity in the developing brain. EurJPaediatrNeurol.2017;21:23-48. PubMed: https://pubmed.ncbi.nlm.nih.gov/27567276/

27. Blanchet M, Prince F, Messier J. Development of postural stability limits: anteroposterior and mediolateral postural adjustment mechanisms do not follow the same maturation process, Human Movement Sci. 2019; 63: 164-171.

PubMed: https://pubmed.ncbi.nlm.nih.gov/25168735/

28. Definitions and classification of tic disorders. The Tourette Syndrome Classification Study Group. Arch Neurol. 1993; 50: 1013-1016. PubMed: https://pubmed.ncbi.nlm.nih.gov/8215958/

29. Goodman WK, Price LH, Rasmussen SA, Mazure C, Fleischmann RL, et al. The yale-brown obsessive-compulsive scale. I. Development use, and reliability. Arch General Psychiatry. 1989; 46: 1006-1011. PubMed: https://pubmed.ncbi.nlm.nih.gov/2684084/

30. Wu G, Chiang JH. The effects of surface compliance on foot pressure in stance. Gait \& Posture. 1996; 4: 122-129.

31. Riach CL, Starkes JL. Velocity of center of pressure excursions as an indicator of postural control systems in children. Gait \& Posture. 1994 2: $167-72$

32. Van Wegen EEH, Van Emmerik REA, Riccio GE, et al. Postural orientation: age-related changes in variability and time-to-boundary. Hum Mov Sci. 2002; 21: 61-84.

PubMed: https://pubmed.ncbi.nlm.nih.gov/11983434/

33. Newton RA. Validity of the multi-directional reach test: a practical measure for limits of stability in older adults. J Gerontol A Biol Sci Med Sci. 2001; 56: 248-52.

PubMed: https://pubmed.ncbi.nlm.nih.gov/11283199/

34. Horak FB, Macpherson JM. Balance orientation and equilibrium, (Section 12, Chapter 7). in: J. Shepard, L. Rowell (Eds.), Handbook of physiology. Exercise: Regulation and Integration of Multiple Systems, Oxford University Press. NY. 1996; 255-292.

35. Cox JH, Seri S, Cavanna AE. Sensory aspects of Tourette syndrome, Neurosci Biobehav Rev. 2018; 88: 170-176.

PubMed: https://pubmed.ncbi.nlm.nih.gov/29559228/

36. Sutherland Owens AN, Miguel EC, Swerdlow NR. Sensory Gating Scales and Premonitory Urges in Tourette Syndrome. Scientific World J. 2011; 22: 736-741.

PubMed: https://pubmed.ncbi.nlm.nih.gov/21442151/

37. Jacobs JV, Horak FB. Abnormal proprioceptive-motor integration contributes to hypometric postural responses of subjects with Parkinson's disease. Neuroscience. 2006; 141: 999-1009. PubMed: https://pubmed.ncbi.nlm.nih.gov/16713110/

38. Orth M, Münchau A. Transcranial magnetic stimulation studies of sensorimotor networks in Tourette syndrome. Behav Neurol. 2013; 27: 57-64.

PubMed: https://pubmed.ncbi.nlm.nih.gov/23187144/

39. Desmurget M, Gaveau V, Vindras P, Turner RS, Broussolle E, et al On-line motor control in patients with Parkinson's disease. Brain. 2004; 127: $1755-1773$

40. Adamovich SV, Berkinblit MB, Hening W, Sage J, Poizner H. The interaction of visual and proprioceptive inputs in pointing to actual and remembered targets in Parkinson's disease. Neuroscience. 2001; 104: 1027-1041.

PubMed: https://pubmed.ncbi.nlm.nih.gov/11457588/

41. Kirshenbaum N, Riach CL, Starkes JL. Non-linear development of postural control and strategy use in young children: a longitudinal study. Experimental Brain Res. 2001; 40: 420-431. PubMed: https://pubmed.ncbi.nlm.nih.gov/11685395/

42. Farhim CF, Yoon U, Das S, Lyttelton O, Chen J. Somatosensorymotor bodily representation cortical thinning in Tourette: Effects of tic severity. Age and Gender. Cortex. 2010; 46: 750-760.

43. Hong KE, Ock SM, Kang MH, Kim CE, Bae JN, et al. The segmented regional volumes of the cerebrum and cerebellum in boys with Tourette syndrome. J Korean Med Sci. 2002; 17: 530-536.

PubMed: https://www.ncbi.nlm.nih.gov/pmc/articles/PMC3054894/

44. Miller AM, Bansal R, Hao X, Sanchez-Pena JP, Sobel LJ, et al. Enlargement of Thalamic Nuclei in Tourette Syndrome. Arc General Psychiatry. 2010; 67: 955-964.

PubMed: https://pubmed.ncbi.nlm.nih.gov/20819989/

45. Peterson BS. Neuroimaging studies of Tourette syndrome: a decade of progress. Advances Neurol. 2001; 85: 179-196. PubMed: https://pubmed.ncbi.nlm.nih.gov/11530427/

46. Sowell ER, Kan E, Yoshii J, Thompson PM, Bansal R, et al. Thinning of sensorimotor cortices in children with Tourette syndrome. Nat Neurosci. 2008; 11: 637-639. PubMed: https://pubmed.ncbi.nlm.nih.gov/18488025/

47. Franzkowiak S, Pollok B, Biermann-Ruben K, Südmeyer M, Paszek J, et al. Motor-Cortical Interaction in Gilles de la Tourette Syndrome. PLoS One. 2012; 7: e27850. PubMed: https://pubmed.ncbi.nlm.nih.gov/22238571/

48. Cohen AJ, Leckman JF. Sensory phenomena associated with Gilles de la Tourette's syndrome. J Clin Psychiatry. 1992; 53: 319-323. PubMed: https://pubmed.ncbi.nlm.nih.gov/1517194/

49. Evers RA, van de Wetering BJ. A treatment model for motor tics based on a specific tension-reduction technique. J Behav Ther Exp Psychiatry. 1994; 25: 255-260.

PubMed: https://pubmed.ncbi.nlm.nih.gov/7852608/

50. Szejko N, Jakubczyk A, Dunalska A, Janik P. Dystonic tics in patients with Gilles de la Tourette syndrome. Neurol Neurochir Pol. 2019; 53: 335-340. PubMed: https://pubmed.ncbi.nlm.nih.gov/31621889/ 